\title{
Modeling of the Contact Center Agent's Work
}

\author{
Erik Chromy and Matej Kavacky
}

\begin{abstract}
The paper deals with the optimal sizing of the contact center. The most important part of the contact centers are their agents, therefore the modeling of their working time is also important. In the case of parameter which describes the number of handled calls during the peak hour also the other factors should be taken into account, such as time for hygienic break and time for other activities after serving of the customer. Such modified parameter can be used in Erlang $C$ equation for calculation of optimal number of the contact center agents.
\end{abstract}

Keywords-Call center, Erlang C, Quality of Service.

\section{INTRODUCTION}

The contact center is dynamic technical system designed for efficient interconnection of users with request for service with operator or with systems capable of meet those needs. The principle is as follows - calling customer should be handled by one or more agents of the contact center. Every agent is trained and supported by corresponding informationtelecommunication system [1]-[7].

In terms of commercial success of the contact center operators, it is important to serve customers as soon as possible and give them as much information. The main objective is to avoid that the calling customer will lost patience by long waiting and thereby avoid any unnecessary loss of interest of client for service. Contact centers are widely used in many areas: telecom operators, banking, insurance, government, education, health, tourism, etc.

The contact center consists of multiple technical components, such as:

- Private Branch eXchange (PBX), with Automatic Call Distribution (ACD) function,

- System management - system for evaluation of handled calls and monitoring of the contact center operation,

- Interactive Voice Response (IVR) [8],

- Computer Telephony Integration (CTI) [9],

- Recording system - not only for recording of the speech interaction, but also recording of agent's screen sequence during serving of the customer,

- Campaign manager - generates outbound calls based on customer requirement and also allows to make calling campaigns,

This article was created with the support of the Ministry of Education, Science, Research and Sport of the Slovak Republic within the KEGA agency project - 007STU-4/2016 named: Progressive educational methods in the field of telecommunications multiservice networks.

E. Chromy is with the Slovak University of Technology, Faculty of Electrical Engineering and Information Technology, Departement of Telecommunications, Ilkovicova 3, 81219 Bratislava, Slovakia (e-mail: chromy@ut.fei.stuba.sk).

M. Kavacky is with the Slovak University of Technology, Faculty of Electrical Engineering and Information Technology, Departement of Telecommunications, Ilkovicova 3, 81219 Bratislava, Slovakia (e-mail: kavacky@ut.fei.stuba.sk).

Manuscript received July 22, 2016, revised September 9, 2016

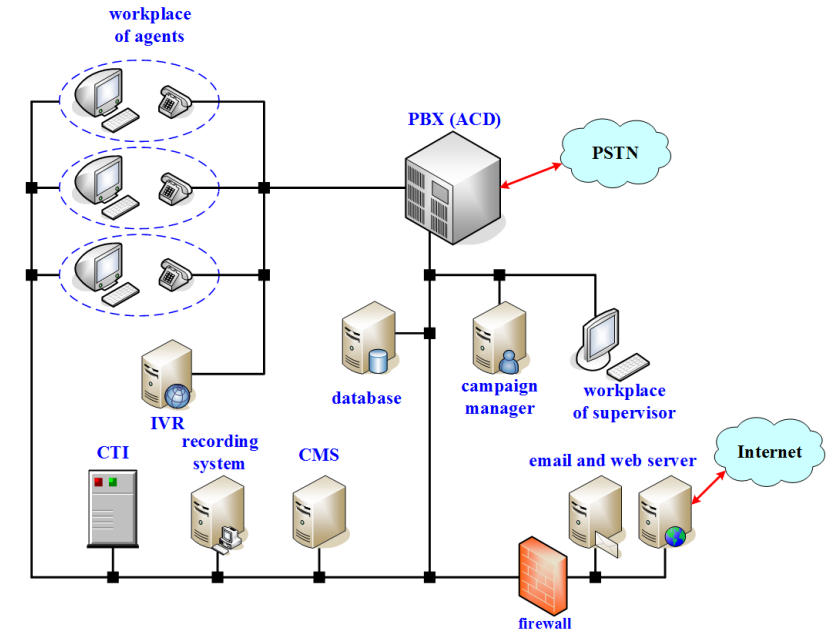

Fig. 1. Technical components of contact center

- Workplace of agent and supervisor,

- email server and

- web server.

\section{Automatic Call Distribution}

$\mathrm{ACD}$ is the basic program equipment necessary for contact center operation. It offers sophisticated and various functions of call routing, creation of queues and control of agent's productivity.

The task of call routing is to interconnect the calling customer with agent. Multiple important parameters should be taken into account for call routing:

- service request of the calling customer,

- profile of the agent,

- state of the agent (busy or idle),

- state of the waiting queue (number of waiting callers, average waiting time),

- system load in real-time and

- alternative resource (in case of overload).

\section{A. Waiting Queue}

The contact center represents the efficient resource usage. Therefore it is necessary to count with the situation, that in the time of call no suitable idle agent is available for the caller.

The PBX enqueues incoming calls. The required service is determined (choice of the caller in the IVR) and based on this the call is routed tothe queue for identified service. Call in the queue behaves according FIFO (First In First Out) rule. The special case occurs when the caller is marked as special. In this case the call is placed to the beginning of the queue.

Calls remain in the queue until: 
- agent of the called group will become idle, or

- expiration of the optional time interval intended for caller waiting (set by manager of the contact center), or

- cancellation of call by the caller.

\section{Qualitative Parameters of the Contact CENTER}

Quality of Services (QoS) parameters important from customer's view:

- time spent for waiting for available agent,

- maximal waiting time for available agent,

- service time of request (communication with agent).

For the customer it is also important:

- to be handled by qualified agent,

- to be handled by one agent (call transfer between multiple agents is not suitable solution).

The setting of optimal number of agents is the important task for contact center manager. Therefore, he should to determine the number of agents such that customers will be satisfied with service and at the same time agents will serve the maximum customers during working time.

Following QoS parameters are important for determining the optimal number of agents [11] from the contact center manager point of view:

- the average number of calls during busy hour,

- the average number of calls waiting in the queue,

- the average waiting time of customer in the queue [10], [12], [14], [15],

- the average service time of customer by agent (this value should be decreased during contact center operation) [13],

- set of maximum waiting time of the customer for serving by agent,

- determine of percentage of incoming calls that should be connected with agent within specified time,

- the probability that all agents are occupied when customer is calling,

- the agent utilization (for determining the number of minutes when agent is active during busy hour).

\section{Estimation of the Optimal Number of Agents THROUGH ERLANG C EQUATION}

We can assume following parameters:

- upper bound for request enqueue: $P_{C}$ (manager will set the probability when all agents will be occupied),

- number of requests the agent can serve during busy hour: $\mu$,

- number of requests that are output of IVR system and are entering the service process: $\lambda$.
Erlang C equation [16]-[20] can be then stated in following form:

$$
P_{C}\left(m, \frac{\lambda}{\mu}\right)=\frac{\frac{m\left(\frac{\lambda}{\mu}\right)^{m}}{m !\left(m-\frac{\lambda}{\mu}\right)}}{\sum_{i=0}^{m-1} \frac{\left(\frac{\lambda}{\mu}\right)^{i}}{i !}+\frac{m\left(\frac{\lambda}{\mu}\right)^{m}}{m !\left(m-\frac{\lambda}{\mu}\right)}} .
$$

From this equation (1) we can calculate the final number of agents $m$ by iteration.

The number of agents $m$ can be calculated also through equation (2) if another parameters are defined:

- Grade of Service (GoS): percentage of requests received for serving by agent until defined time AWT (Acceptable Waiting Time),

- AWT: maximal time which the request can spent in queue for available agent for defined GoS percentage.

$$
G o S=1-P_{C} \cdot e^{-\mu\left(m-\frac{\lambda}{\mu}\right) A W T} .
$$

By modification of equation (2) we have equation (3), which states the required number of contact center agents:

$$
m=\frac{A W T \cdot \lambda-\ln \left(\frac{1-G o S}{P_{C}}\right)}{A W T \cdot \mu} .
$$

Based on such stated number of agents $m$ it is possible to calculate another QoS parameters of the contact center from Erlang $\mathrm{C}$ equation:

- occupancy of one agent in serving group:

$$
\rho=\frac{\lambda}{m \cdot \mu},
$$

- average waiting time in serving group queue:

$$
W=\frac{P_{C}}{\mu\left(m-\frac{\lambda}{\mu}\right)},
$$

- average number requests in service time queue:

$$
Q=\lambda . W=\frac{\lambda}{\mu\left(m-\frac{\lambda}{\mu}\right)} \cdot P_{C},
$$

- average number of requests in service time system:

$$
N=\frac{\lambda}{\mu}+Q=\frac{\lambda}{\mu}+\frac{\lambda}{\mu\left(m-\frac{\lambda}{\mu}\right)} \cdot P_{C},
$$

- average time which request will spent in service time system: 


$$
T=\frac{N}{\lambda}=\frac{1}{\mu}+W=\frac{1}{\mu}+\frac{P_{C}}{\mu\left(m-\frac{\lambda}{\mu}\right)} .
$$

\section{Modeling of the Working Time of Contact CEnTER Agent}

In the case of activity of contact center agent we can think about two notions: idle time and administrative work.

The notion idle time in the contact center system represents time dedicated for example for hygienic break. The contact center manager can set $I_{T}$ minutes of working hour for such idle time. Idle times have also impact on contact center sizing. The modeling of the idle time can be done in two ways:

\section{A. Idle Time - Shortening of the Average Service Time}

If we want to preserve the original value of parameter $\mu$ (as we assume in chapter 4), representing average number of handled calls during busy hour, then we have to change average service time required for serving of one request (based on given parameter $I_{T}$ - in minutes).

Then for average service rate of one request the following equation must be valid:

$$
\frac{1}{\mu_{I_{T}}}=\frac{1}{\mu}-\frac{\frac{I_{T}}{60}}{\mu}=\frac{60-I_{T}}{60 \cdot \mu},
$$

where $1 / \mu_{I_{T}}$ represents average time (in hours) in which the request must be handled while the original value of parameter $\mu$ must be preserved. It means faster service time and also following inequality must apply:

$$
\frac{1}{\mu_{I_{T}}}<\frac{1}{\mu} .
$$

If the shortening of average service time is not suitable, then the number of handled calls during busy hour must be modified (decreased).

\section{B. Idle Time - Handled Calls Decreasing}

It is necessary to decrease the number of requests the one agent can serve during busy hour. It leads to increased number of agents required for serving calls. In this way the average service time of one request is not changed.

If we will assume with idle times of agent, then we must use new parameter $1 / \mu_{I_{T}}$ in our calculations:

$$
\mu_{I_{T}}=\frac{60-I_{T}}{\frac{60}{\mu}}=\frac{\mu\left(60-I_{T}\right)}{60} .
$$

This way of calculation of optimal number of agents in contact center is preferable, because the service time is not shortened.

\section{Administrative Work}

The notion administrative work in contact center system represents activities the agent must done after every handled call. It means that customer can have request during call which the agent must handle right after the call (e.g. search for invoice or detailed record list, send e-mail, etc.). But such activity has negative impact on number of handled calls during work time of agent.

It is obvious that such activity is not required after every call, but after only percentage of calls. Therefore in the modeling of contact center processes we should also this parameter take into account. This parameter will be denoted as $a_{W}$. We must also set how much time (in minutes) the agent require for such activity in average. This parameter will be denoted as $T_{a_{W}}$ (in minutes). Assumption of administrative work means that the average service time of one request for one agent will be extended (according to values of parameters $a_{W}$ and $T_{a_{W}}$ ).

The parameter $T_{a_{W}}$ can have values in the range $<0,1>$.

- 0: it means that administrative work is not assumed,

- 1 : it means that administrative work is assumed for every call.

Therefore, if we assume administrative work it is necessary to modify (before modeling) the original value of parameter $\mu$ (resp. 1/ $)$, which represents number of handled calls during busy hour and in calculations we must use new parameter $\mu_{a_{W}}$ :

$$
\frac{1}{\mu_{a_{W}}}=\frac{1+\frac{\mu \cdot a_{W} \cdot T_{a_{W}}}{60}}{\mu},
$$

where, $1 / \mu_{a_{W}}$ represents average service time of call (in hours) while assuming parameters $a_{W}$ and $T_{a_{W}}$.

Then the following inequality will be valid:

$$
\frac{1}{\mu_{a_{W}}}>\frac{1}{\mu} \text {. }
$$

The inequality (13) leads to increased number of agents required for handling calls.

Based on above assumptions we can write the final equation (14), which takes into account the idle times and administrative work of agent $\mu_{I_{T}+a_{W}}$ and modifies the original parameter $\mu$, (resp. $1 / \mu$ ) into following form:

$$
\begin{aligned}
& \mu_{I_{T}+a_{W}}=\frac{\frac{\mu}{1+\frac{\mu \cdot a_{W} \cdot T_{a_{W}}}{60}} \cdot\left(60-I_{T}\right)}{60}= \\
& =\frac{\mu\left(60-I_{T}\right)}{60+\mu \cdot a_{W} \cdot T_{a_{W}}} .
\end{aligned}
$$

Also the following inequality will be valid:

$$
\mu>\mu_{I_{T}+a_{W}} .
$$

From equation (15) we can see that if we will assume the idle times and administrative work of agent, the average number of handled calls during busy hour will be lower than in the case when not assuming the idle times and administrative 
work. Such assumption and the use of new parameter $\mu_{I_{T}+a_{W}}$ in calculations of the optimal number of agents leads to increased number of the required agents.

If we will assume the idle time and administrative work, then we can use Erlang $\mathrm{C}$ equation and equation for calculation of required number of agents based on GoS parameter in following modifications:

- determine the optimal number of agents using the Erlang $\mathrm{C}$ equation:

$$
P_{C}\left(m, \frac{\lambda}{\mu_{I_{T}+a_{W}}}\right)=\frac{\frac{m\left(\frac{\lambda}{\mu_{I_{T}+a_{W}}}\right)^{m}}{m !\left(m-\frac{\lambda}{\mu_{I_{T}+a_{W}}}\right)}}{\sum_{i=0}^{m-1} \frac{\lambda\left(\frac{\lambda}{\mu_{I_{T}+a_{W}}}\right)^{i}}{i !}+\frac{m\left(\frac{\lambda}{\mu_{I_{T}+a_{W}}}\right)^{m}}{m !\left(m-\frac{\lambda}{\mu_{I_{T}+a_{W}}}\right)}},
$$

- determine the optimal number of agents using Erlang C equation when the parameter GoS is known:

$$
m=\frac{A W T \cdot \lambda-\ln \left(\frac{1-G o S}{P_{C}}\right)}{A W T \cdot \mu_{I_{T}+a_{W}}} .
$$

\section{CONCLUSION}

In the paper we presented possibilities of modeling of working time of the contact center agent. The parameter of service time of customer we have extended with two more parameters. We took into account the idle time and time for administrative work. Based on this consideration we derived the modification of Erlang $\mathrm{C}$ equation for determine the optimal number of the contact center agents.

\section{REFERENCES}

[1] I. Baronak and E. Chromy, Call Center and its Evolution, 2nd International Conference on Emerging Telecommunications Technologies and Applications and the 4th Conference on Virtual University, Kosice, Slovak Republic, 2003, pp. 85-88, ISBN 80-89066-67-4

[2] I. Baronak and E. Chromy, The Technology of Call Center Raises the Value of the Society. 26th International Conference Telecommunications and Signal Processing, Brno, Czech Republic, 2003, pp. 56-60, ISBN 80-214-2433-8

[3] E. Chromy and I. Baronak, Application of Call Center. 10th International Scientific Conference COFAX-TELEKOMUNIKACIE 2004, Bratislava, Slovak Republic, 2004, pp. 129-136, ISBN 80-967019-6-7

[4] I. Baronak and E. Chromy, Call center client application interface. 27th International Conference Telecommunications and Signal Processing, Brno, Czech Republic, 2004, pp. 30-37, ISBN 80-214-2684-5

[5] I. Baronak and E. Chromy, Contact center part of modern communication infrastructure. 3rd International Conference on Emerging Telecommunications Technologies and Applications and the Workshop Organisation and Management of Scientific, Technology and Industria Parks, Kosice, Slovak Republic, 2004, pp. 79-83, ISBN 80-89066-85-2

[6] E. Chromy and I. Baronak, Contribution of call center to regional development. 5th EURASIP Conference focused on Speech and Image Processing, Multimedia Communications and Services, Smolenice, Slovak Republic, 2005, pp. 212-215, ISBN 80-227-2257-X

[7] G. Koole, Call Center Mathematics: A Scientific Method for Understanding and Improving Contact Centers. Departement of Mathematics, Vrije Universiteit, Amsterdam, January, 2007

[8] I. Baronak and E. Chromy, Interactive voice response as a part of call center. 28th International Conference Telecommunications and Signal Processing, Brno, Czech Republic, 2005, pp. 20-23, ISBN 80-214-29720
[9] E. Chromy and I. Baronak, Computer Telephony Integration as a part of Call Center. 6th RTT Conference focused on Research in Telecommunication Technology, RTT 2005, Hradec nad Moravici, Czech Republic, 2005, pp. 204 206, ISBN 80-248-0897-8

[10] G. Koole, B. Nielsen and T. Nielsen, First in line waiting times as a tool for analyzing queueing system. Operations Research, Vol. 80, No. 5, 2012, pp. 1258-1266, DOI: 10.1287/opre.1120.1089

[11] N. Gans, H. Shen, Y-P. Zhou, N. Korolev, A. McCord and H Ristock, Parametric Stochastic Programming Models for Call-Center Workforce Scheduling. Manufacturing and Service Operations Management, Vol. 17, No. 4, 2015, pp. 571-588, DOI: $10.1287 / \mathrm{msom} .2015 .0546$

[12] B. Legros, O. Jouini and G. Koole, Adaptive Threshold Policies for Multi-Channel Call Centers. IEE Transactions, Vol. 47, No. 4, 2015, pp. 414-430, ISSN: 0740-817X, DOI: 10.1080/0740817X.2014.928965

[13] A. Weerasinghe, Optimal service rate perturbations of many server queues in heavy traffic. Queueing Systems, Vol. 79, No. 3-4 2015, Springer US, pp. 321-363, ISSN: 0257-0130, DOI: 10.1007/s11134-0149423-9

[14] W. Chan, G. Koole and P. Ecuyer, Dynamic Call Center Routing Policies using Call Waiting and Agent Idle Times. Manufacturing and Service Operations Management, Vol. 16, No. 4, 2014, pp. 544-560, DOI: 10.1287/msom.2014.0493

[15] G. Koole, B. Nielsen and T. Nielsen, T.: Optimization of overflow policies in call centers. Probability in the Engineering and Informational Sciences, Cambridge University Press, Vol. 29, No. 3, 2015, pp. 461471, DOI: 10.1017/S0269964815000091

[16] E. Chromy, T. Misuth and M. Kavacky, Erlang C Formula and Its Use In the Call Centers. Journal AEEE - Information and Communication Technologies and Services, Vol. 9, No. 1, pp. 7-13, March 2011, ISSN 1804-3119

[17] E. Chromy, J. Diezka, M. Kovacik and M. Kavacky, Traffic Analysis in Contact Centers. The 11th International Conference KTTO 2011, June 22-24, Szczyrk, Poland, 2011, pp. 19-24, ISBN 978-80-248-2399-7

[18] E. Chromy, T. Misuth and A. Weber, Application of Erlang Formulae in Next Generation Networks. International Journal of Computer Network and Information Security (IJCNIS), Vol. 4, No. 1, February 2012, MECS Publisher, 2012, pp. 59-66, ISSN: 2074-9090 (Print), ISSN: 2074-9104 (Online)

[19] O. Jouini, G. Koole and A. Roubos, Performance Indicators for Call Centers with Impatience. IEE Transactions, Vol. 45, No. 3, 2013, pp. 341-354, ISSN: 0740-817X, DOI: 10.1080/0740817X.2012.712241

[20] J. Zan, J. Hasenbein and D. Morton, Asymptotically optimal staffing of service systems with joint QoS constraints. Queueing Systems, Vol. 78, No. 4, 2014, Springer US, pp. 359-386, ISSN: 0257-0130, DOI $10.1007 / \mathrm{s} 11134-014-9406-\mathrm{x}$

Erik Chromy was born in Velky Krtis, Slovakia, in 1981. He received the M.Sc. degree in telecommunications in 2005 from Faculty of Electrical Engineering and Information Technology of Slovak University of Technology (FEI STU) Bratislava. In 2007 he submitted PhD work. His scientific research is focused on queueing systems, contact center and admission control methods. Nowadays he works as assistant professor at the Institute of Telecommunications of FEI STU Bratislava.

Matej Kavacky was born in Nitra, Slovakia, in 1979. He received the M.Sc. degree in telecommunications in 2004 from Faculty of Electrical Engineering and Information Technology of Slovak University of Technology in Bratislava (FEI STU). In 2006 he submitted PhD. work Quality of Service in Broadband Networks. His scientific research is focused on admission control methods Nowadays he works as assistant professor at the Institute of Telecommunications of FEI STU Bratislava. 